\title{
EBV-HLH children with reductions in CD4+ $T$ cells and excessive activation of CD8+ $T$ cells
}

\author{
Cheng Yang ${ }^{1,4}$, Xiujuan Zhu ${ }^{2,4}$, Ting Zhang ${ }^{3,4}$ and Qing Ye ${ }^{1}$
}

BACKGROUND: In this report, we analyzed the number and activation profiles of $\mathrm{T}$ cells in Epstein-Barr virus-associated hemophagocytic lymphohistiocytosis (EBV-HLH) patients and assessed their value for the diagnosis of EBV-HLH.

METHODS: We compared the quantity of CD4+ $T$ cells and CD8+ T cells in peripheral blood among HLH patients, a sepsis group, and healthy controls. We then analyzed variations in the activation marker in CD4+ T and CD8+ T cells.

RESULTS: The percentage of CD4+ $T$ cells decreased in the EBV-HLH patients when compared with healthy controls, which induced significant differences in the ratio of CD4+ $T$ cells/CD8+ T cells. The percentage of CD4+HLA-DR+ T cells, CD8+CD69+ T cells, CD8+HLA-DR+ T cells, and CD8+HLA$\mathrm{DR}+\mathrm{T}$ cells/CD4+CD25+ T cells increased in EBV-HLH patients when compared with that in healthy controls. Compared with EBV-HLH survivors, the median percentage of CD4+CD25+ T cells (7.9 vs. 3.0) were decreased, but the ratios of CD8+HLA$\mathrm{DR}+\mathrm{T}$ cells/CD4+CD25+T cells (6.3 vs. 20.3) were increased in the dead patients.

CONCLUSIONS: There was a reduction in CD4+ $T$ cells and abnormal activation of CD8+ T cells in the EBV-HLH patients. The percentage of $C D 4+C D 25+T$ cells and the ratios of CD8+HLA-DR+ T cells/CD4+CD25+ T cells in EBV-HLH patients had a relation to the prognosis of the disease.

$\mathbf{H}$ emophagocytic lymphohistiocytosis is a rare hyperinflammatory syndrome that is fatal (1). There are two forms of HLH: primary and secondary. Primary HLH is an autosomal-recessive disease that exhibits genetic defects. Secondary HLH is associated with infection, and EBV is one of the most frequent triggers of infection-associated HLH (2). HLH is characterized by a combination of signs, including persistent fever, splenomegaly with cytopenia, hypertriglyceridemia, hypofibrinogenemia, and an elevated serum ferritin level (3). An HLH study group of the histiocyte society developed a set of diagnostic criteria for HLH (4), and the diagnosis mainly depends on clinical and laboratory findings exhibited by the affected patients. Although these findings used to diagnose HLH were nonspecific, they often lead to delayed treatment $(5,6)$. Despite attempts to differentiate HLH from sepsis patients, the symptomatic presentations overlap highly (7). Timely diagnosis is significant for starting therapy before the damage becomes irreversible (8). Therefore, specific diagnostic indices are urgently needed.

Therefore, in this article, we focused on expression of $\mathrm{T}$ cells activation markers and evaluated the usefulness of these activation markers on the surface of $\mathrm{T}$ cells for differential diagnosis of HLH disease.

\section{METHODS}

\section{Participants}

This prospective study was conducted from January 2013 to December 2015 at the Children's Hospital of Zhejiang University School of Medicine. Ethics approval was obtained from the Institutional Review Board of Zhejiang University (Approval number: 2013015). All participants provided informed consent. Patients who were recruited had documented EBV infections and met the HLH-2004 protocol (4). All the patients provided detailed medical histories and underwent physical and laboratory examinations. Those participants suffering from other diseases that may have interfered with this study were excluded. Sepsis patients and healthy children who matched in age and gender were also included as control.

\section{Measurement of Activated T-Cell Subsets}

Blood was collected directly and resuspended at a concentration of $1 \times 10^{6}$ cells $/ \mathrm{ml}$ in RPMI 1640 medium (Biomed, Lublin, Poland). Next, the blood was incubated in 24-well flat-bottomed plates at a final volume of $0.2 \mathrm{ml}$ (per well) and was collected after $24 \mathrm{~h}$ at $37^{\circ} \mathrm{C}$, $5 \% \mathrm{CO}_{2}$. Flow cytometric immunophenotyping was performed using flow cytometry with double staining (BD Biosciences, San Jose, CA). The main reagents used in this study were as follows: CD3-FITC, CD8-PreCP, CD4-APC, CD25-PE, CD69-PE, HLA-DR-PE, and FACS Lysing Solution. Then, $100 \mu \mathrm{l}$ of blood was mixed and incubated for $30 \mathrm{~min}$ at room temperature with appropriate quantities of antibodies or controls. After a short period of incubation and rinsing, the samples were fixed with $1 \%$ paraformaldehyde and analyzed with flow cytometry. The data were collected on a BD FACSCanto II (BD Biosciences, San Jose, CA, USA) and analyzed with Cell Quest software (BD Biosciences, San Jose, CA, USA).

\section{Measurement of natural killer (NK) cell Activity In Vitro}

The lytic activities of NK cells were measured with an MTT-based assay. Approximately $5 \times 10^{3}$ K562 cells were plated on round-

${ }^{1}$ Zhejiang Key Laboratory for Neonatal Diseases, Clinical Laboratory, The Children's Hospital of Zhejiang University School of Medicine, Hangzhou, China; ${ }^{2}$ Department of Nephrology, The Children's Hospital of Zhejiang University School of Medicine, Hangzhou, Zhejiang, China; ${ }^{3}$ College of Medical Technology, Zhejiang Chinese Medical University, Hangzhou, Zhejiang, China. Correspondence: Qing Ye (yeqingkaoyan@163.com)

${ }^{4}$ These authors contributed equally to this work.

Received 1 May 2017; accepted 20 June 2017; advance online publication 16 August 2017. doi:10.1038/pr.2017.178 
bottom 96-well titer plates. The NK cells isolated from peripheral blood of each EBV-HLH child were added into each well to yield effector cells to target cells (E:T) at ratios of 10:1 in a final volume of $0.2 \mathrm{ml}$, in triplicate. After incubation for $4 \mathrm{~h}$ at $37^{\circ} \mathrm{C}$ and $5 \% \mathrm{CO}_{2}$, $10 \mu \mathrm{l} \mathrm{MTT}(5 \mathrm{mg} / \mathrm{ml})$ was added to each well for an additional $4 \mathrm{~h}$. The plates were then centrifuged at $2,000 \mathrm{~g}$ for $15 \mathrm{~min}$ and $100 \mu \mathrm{l}$ of the supernatant was replaced with $100 \mu 10 \%$ sodium dodecyl sulphate. The plates were incubated overnight to completely dissolve the pellet. Optical density (OD) was analyzed at $570 \mathrm{~nm}$ and cytotoxicity was assessed by the following formula: cytotoxicity $(\%)=\left[1-\left(\mathrm{OD}_{\mathrm{E}+\mathrm{T}}-\mathrm{OD}_{\mathrm{E}}\right) / \mathrm{OD}_{\mathrm{T}}\right] \times 100 \%$. Thirty healthy children who matched in age and gender were included as normal control.

\section{Statistical Analysis}

All statistical analyses were performed using SPSS Version 18.0 (PASW Statistics for Windows, Version 18.0, SPSS, Chicago, IL). The Mann-Whitney $U$-test was used to compare the differences in continuous variables. The $\chi^{2}$ test was used to compare the differences in categorical variables. $P<0.05$ was considered to be significant. We used receiver operating characteristic (ROC) curves to assess the diagnostic value, the optimal diagnostic threshold was determined according to Youden's index, and the relative sensitivity and specificity were calculated.

\section{RESULTS}

\section{Characteristics of EBV-HLH Patients}

The study participants included 182 EBV-HLH patients (95 boys and 87 girls with a median age of 2.4 years, range: 1.1-14.2 years). The clinical features of these patients are shown in Figure 1. The cardinal symptoms and signs were fever (99\%). Most of the patients presented characteristic laboratory abnormalities, including elevated ferritin (98\%), thrombocytopenia (91\%), hemophagocytosis (90\%), hepatosplenomegaly (84\%), hypofibrinogenemia (81\%), and neutropenia $(81 \%)$. In addition, $80 \%$ of cases showed signs of hypertriglyceridemia and anemia. Impaired $\mathrm{NK}$ cell cytotoxicity was also a characteristic finding in EBV-HLH patients $(84 \%)$.

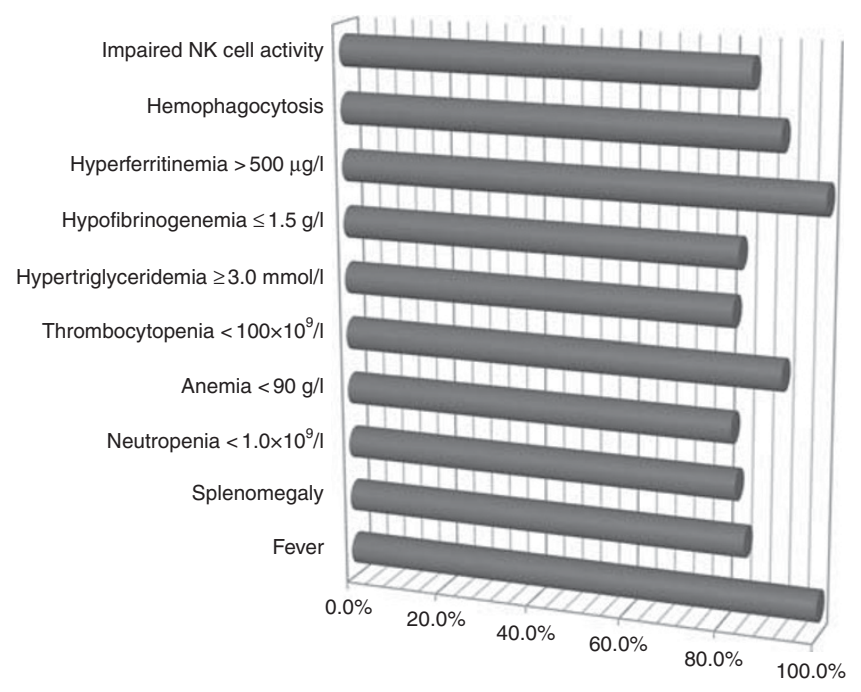

Figure 1. Characteristics of EBV-HLH patients.

\section{T-Cell Subset and its Activation Levels in EBV-HLH Patients}

Our data demonstrated a statistically significant decrease in EBV-HLH patients of the median percentage of CD4+ T cells (21.0 vs. 34.6$)$ and the ratio of $\mathrm{CD} 4+\mathrm{T}$ cells/CD8+ $\mathrm{T}$ cells $(1.0$ vs. 1.5) when compared with the ratios in the control group; however, there was no significant difference in the percentage of CD8+ $\mathrm{T}$ cells. To discover variations of the activation markers on the surface of $\mathrm{T}$ cells, we measured the markers in EBV-HLH patients in comparison with healthy controls (Table 1 and Figures 2 and 3). There were significant differences in the percentage of CD4+HLA-DR+ $\mathrm{T}$ cells, CD8+ CD69+ T cells, and CD8+HLA-DR+ T cells and the ratio of CD8+HLA-DR+ T cells/CD4+CD25+ T cells between the HLH patients and healthy control groups $(P<0.05)$. However, there were no significant differences in the percentage of CD4+CD69+ T cells, CD4+ CD25+ T cells, and $\mathrm{CD} 8+\mathrm{CD} 25+\mathrm{T}$ cells between the two groups $(P>0.05)$. The median percentages of CD4+HLA-DR+ T cells, CD8+ CD69+ T cells, and CD8+HLA-DR+ T cells and the ratio of CD8+HLA-DR+ T cells/CD4+CD25+ T cells (12.9 vs. 5.7, 3.4 vs. $1.2,57.3$ vs. 4.2 , and 10.5 vs. 0.7 , respectively) in HLH patients were significantly elevated when compared with the healthy control group.

\section{Diagnostic Accuracy of the T-Cell Subset and its Activation Markers in EBV-HLH}

To distinguish sepsis from EBV-HLH, a T-cell subset and its activation markers were measured in both EBV-HLH patients and a sepsis group. The percentage of CD4+ T cells, CD4+ CD25+T cells, and CD8+HLA-DR+ T cells, and the ratios of CD4+ T cells/CD8+T cells and CD8+HLA-DR+ T cells/CD4+ CD25+T cells were significantly different between the EBVHLH patients and the sepsis group. When compared with EBV-HLH patients, the median percentages of CD4+ T cells (33.2 vs. 21.0 ), CD4+ T cells/CD8+T cells (1.9 vs. 1.0 ), and CD4+CD25+ T cells (8.3 vs. 5.5) were increased in the sepsis group, but the median percentage of CD8+HLA-DR+T cells (25 vs. 57.3) and the ratios CD8+HLA-DR+ T cells/CD4+ CD25+ T cells (2.9 vs. 10.5) were decreased in the sepsis group when compared with the EBV-HLH patients. As analyzed above, we used ROC curves to assess their diagnostic value for distinguishing sepsis and EBV-HLH. The percentage of CD8+HLA-DR+ T cells and the ratio of CD8+HLA-DR+ $\mathrm{T}$ cells/CD4+CD25+ $\mathrm{T}$ cells were found to be the most efficient indicators (Figure 4). When the percentage of CD8+ HLA-DR+ T cells exceeded $76.44 \%$, the sensitivity was $28.21 \%$ and the specificity was $97.44 \%$. When the ratio of CD8+HLA$\mathrm{DR}+\mathrm{T}$ cells/CD4+CD25+ $\mathrm{T}$ cells exceeded 9.125, its sensitivity was $56.41 \%$ and the specificity was $97.44 \%$.

\section{Predictive Value of T-cell subset and its Activation Levels for the Outcome of Disease}

In order to assess the predictive value of T-cell subset and its activation levels for the outcome of EBV-HLH children, their levels were compared between the EBV-HLH survivors and EBV-HLH dead patients. The result shows that the percentage 


\section{Articles | Yang et al.}

Table 1. T-cell quantity and state of T-cell activation in HLH patients

\begin{tabular}{|c|c|c|c|}
\hline Parameters & Normal control $(n=156)$ & $\mathrm{HLH}(n=182)$ & Sepsis $(n=178)$ \\
\hline $\mathrm{CD} 3+(\%)$ & $64.6(57.9-69.5)$ & $61.4(5.8-564.2)$ & $59.4(22.9-83.6)$ \\
\hline $\mathrm{CD} 3+\mathrm{CD} 4+(\%)$ & $34.6(29.3-46.4)$ & $21.0(1.1-53.1)$ & $33.2(8.9-51.6)$ \\
\hline $\mathrm{CD} 3+\mathrm{CD} 4+/ \mathrm{CD} 3+\mathrm{CD} 8+$ & $1.5(1.1-2.8)$ & $1.0(0.1-5.2)$ & $1.9(0.3-5.0)$ \\
\hline $\mathrm{CD} 3+\mathrm{CD} 4+\mathrm{CD} 25+(\%)$ & $6.3(3.9-8.4)$ & $5.5(0.2-18.3)$ & $8.3(1.5-25.8)$ \\
\hline $\mathrm{CD} 3+\mathrm{CD} 4+\mathrm{HLA}-\mathrm{DR}+(\%)$ & $5.7(3.4-8.0)$ & $12.9(0.2-70.3)$ & $7.7(0.04-73.1)$ \\
\hline $\mathrm{CD} 3+\mathrm{CD} 8+\mathrm{CD} 25+(\%)$ & $1.2(0.3-2.3)$ & $0.5(0.03-24.5)$ & $0.5(0.03-8.4)$ \\
\hline $\mathrm{CD} 3+\mathrm{CD} 8+\mathrm{CD} 69+(\%)$ & $1.2(0.3-1.9)$ & $3.4(0.2-21.4)$ & $1.5(0.03-28.0)$ \\
\hline $\mathrm{CD} 3+\mathrm{CD} 8+\mathrm{HLA}-\mathrm{DR}+(\%)$ & $4.2(2.9-8.1)$ & $57.3(0.3-94.2)$ & $25.0(1.1-80.2)$ \\
\hline $\mathrm{CD} 3+\mathrm{CD} 8+\mathrm{HLA}-\mathrm{DR}+/ \mathrm{CD} 3+\mathrm{CD} 4+\mathrm{CD} 25+$ & $0.7(0.6-1.0)$ & $10.5(0.03-325.0)$ & $2.9(0.2-24.2)$ \\
\hline
\end{tabular}

Values are the median (range).
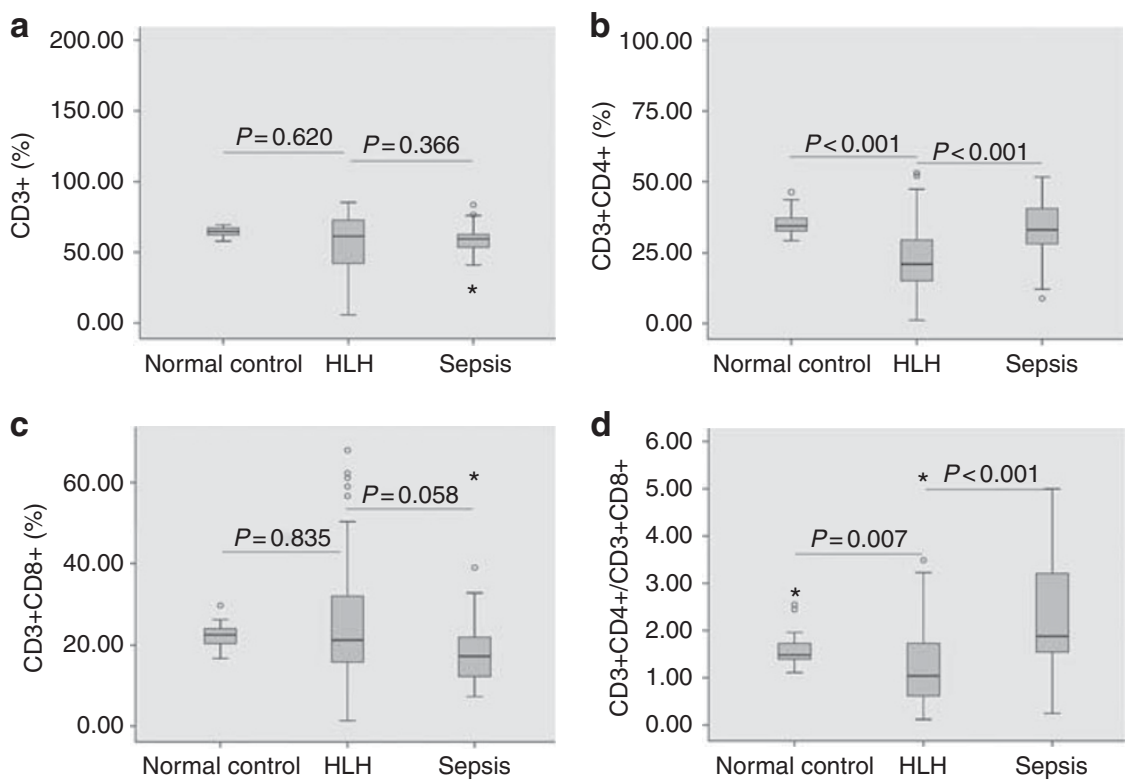

Figure 2. The percentage of T-cell subgroups. The median percentage of CD3+ cells (a), CD4+ T cells (b), and CD8+ T cells (c), and the ratio of CD4+ $\mathrm{T}$ cells/CD8+ T cells (d) in HLH patients, the sepsis group, and healthy controls. The circle and star on behalf of outliers.

of $\mathrm{CD} 4+\mathrm{CD} 25+\mathrm{T}$ cells and the ratios of CD8+HLA-DR+ $\mathrm{T}$ cells/CD4+CD25+T cells were significantly different between them $(P<0.01)$. However, there were no significant differences in the percentage of CD8+HLA-DR+ T cells between the two groups $(P>0.05)$. When compared with EBV-HLH survivors, the median percentage of CD4+CD25+ $\mathrm{T}$ cells (7.9 vs. 3.0) was decreased, but the ratios of CD8+ HLA-DR+ T cells/CD4+CD25+ T cells (6.3 vs. 20.3) were increased in the dead patients (Figure 5).

\section{DISCUSSION}

EBV-HLH is a form of acquired, infection-related HLH that typically represents a fulminant presentation of an acute EBV infection with a $30-50 \%$ mortality rate (9). It has even been suggested that treatment should be started based on a strong clinical suspicion of HLH before overwhelming disease activity occurs, which usually leads to irreversible damage in some patients (10). Currently, a diagnosis of HLH disease mainly depends on clinical symptoms and laboratory tests. We investigated 182 EBV-HLH patients and found that $81 \%$ of patients had hypofibrinogenemia, 91\% had thrombocytopenia, $84 \%$ had hepatosplenomegaly, $80 \%$ of cases showed hypertriglyceridemia, and $81 \%$ revealed neutropenia. It can be seen that the patient's symptoms cannot meet all of the diagnostic criteria and did not appear at the same time. In addition, these symptoms were nonspecific, which led to an indefinite diagnosis (11). Thus, reaching a diagnosis of EBV-HLH is challenging.

HLH is a disorder of the regulatory pathways of immune/ inflammatory responses $(11,12)$. It is currently believed that 


\section{Activation profiles of $T$ cells in EBV-HLH $\quad$ Articles}
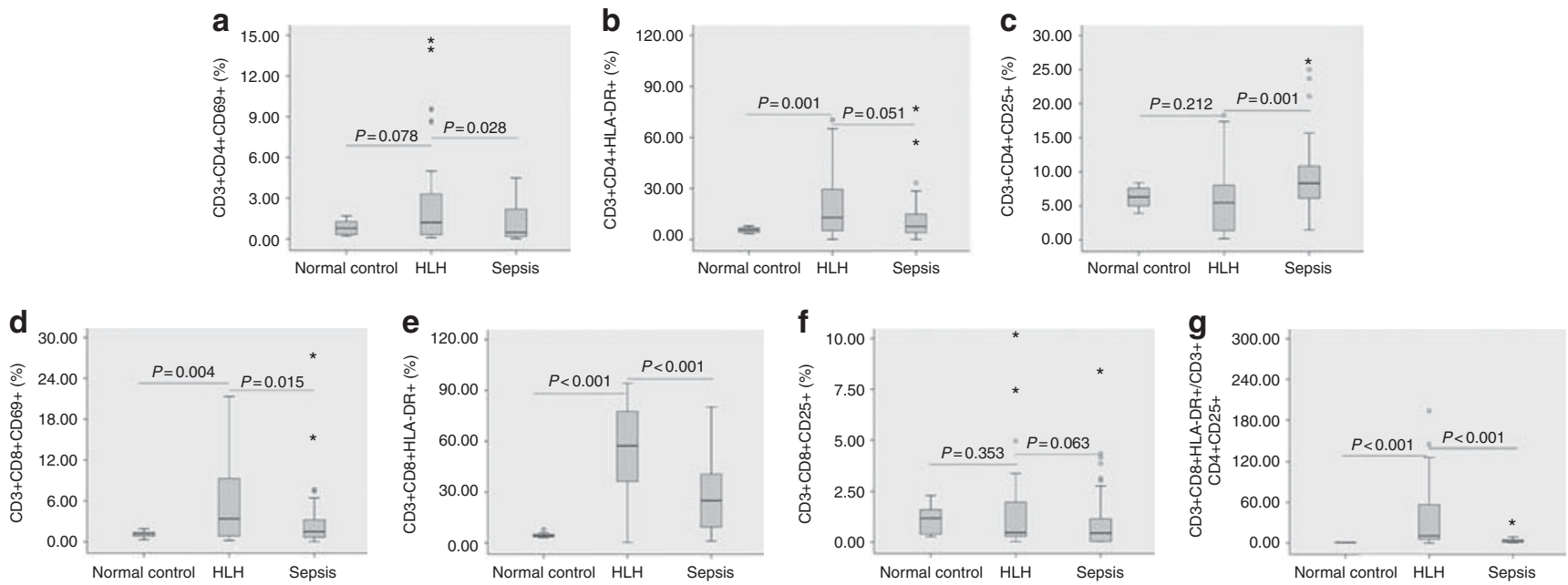

Figure 3. The expressions of CD69, CD25, and HLA-DR on the surface of CD4+ $T$ cells and CD8+ $T$ cells. The percentage of CD4+CD69+ $T$ cells (a), CD4+HLA-DR+ T cells (b), CD4+ CD25+ T cells (c), CD8+ CD69+ T cells (d), CD8+HLA-DR+ T cells, (e) and CD8+CD25+ T cells (f), and the ratio of CD8+HLA-DR+ T cells/CD4+ CD25+ T cells (g) in HLH patients, the sepsis group, and healthy controls. The circle and star on behalf of outliers.
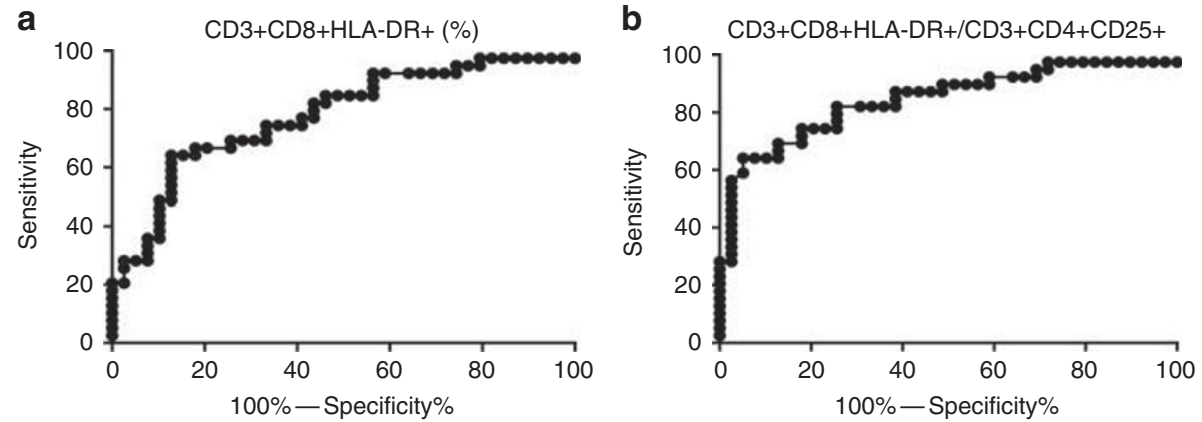

Figure 4. ROC curve for the differential diagnosis values of the percentage of CD8+HLA-DR+ $T$ cells and the ratio of CD8+HLA-DR+ T cells/CD4+ CD25+ T cells in patients with EBV-HLH or sepsis. ROC of CD8+HLA-DR+ T cells (a), with an area under the curve (AUC) of 0.78 and ROC of CD8 + HLA-DR+ T cells/CD4+CD25+ T cells (b), with an AUC of 0.84 . ROC, receiver operating characteristic.
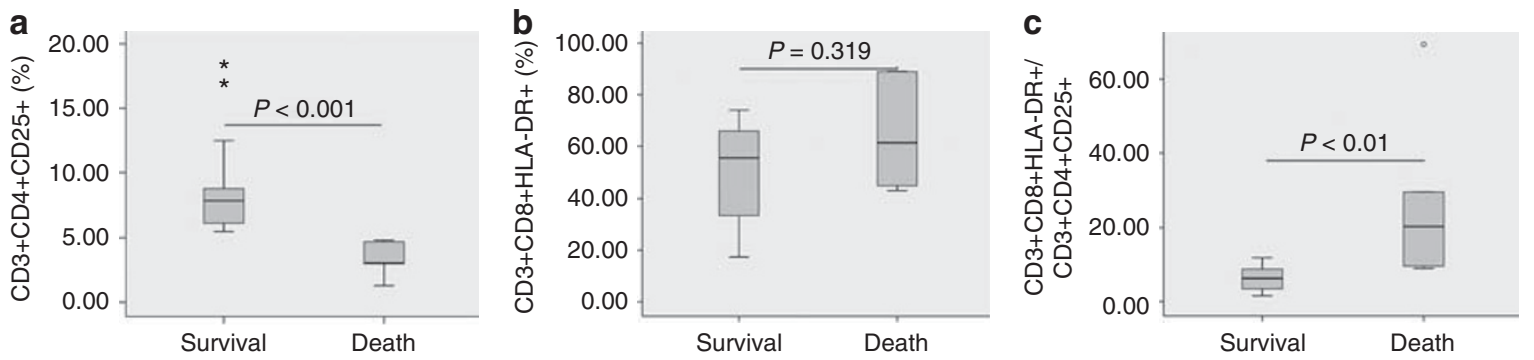

Figure 5. The percentages of CD4+CD25+ $T$ cells and CD8+HLA-DR+ $T$ cells, and the ratios of CD8+HLA-DR+ $T$ cells/CD4+CD25+ $T$ cells in patients with different prognosis. When compared with EBV-HLH survivors, the percentage of CD4+CD25+ T cells (a) was decreased, but the ratios of CD8+ HLA-DR+ T cells/CD4+CD25+ T cells (c) were increased in the dead patients. The percentage of CD8+HLA-DR+ T cells (b) was comparable. The circle and star on behalf of outliers.

high levels of activating cytokines generated by uncontrolled activation of immune cells lead to the death of EBV-HLH patients (13-16). Our previous study found that the levels of interferon- $\gamma$, interleukin (IL)-6, and IL-10 were significantly increased in EBV-HLH patients. These cytokines were down- stream effector molecules and were indirect evidence of activation of $\mathrm{T}$ lymphocytes $(6,17)$. CD69+ antigen markers are involved in activating signal transduction and are an early activation marker that leads to the synthesis of various cytokines. CD25+ antigen, which is a medium-term activation 
indicator, occurs mainly in activated $\mathrm{T}$ cells. HLA/DR+ antigen is expressed in T cells, and it occurs during the later stages of $\mathrm{T}$ lymphocyte and NK cell activation $(6,18)$. To determine $\mathrm{T}$-cell activation levels, we measured these three activation markers in CD4+ $\mathrm{T}$ cells and CD8 $+\mathrm{T}$ cells, respectively. The results showed that there was activation of CD4+ $\mathrm{T}$ cells (high expression of HLA-DR) and CD8+ $\mathrm{T}$ cells (high expression of HLA-DR and CD69). In addition, the numbers of CD8+ $\mathrm{T}$ cells had not changed, and the percentage of CD4+ $\mathrm{T}$ cells decreased in the EBV-HLH patients when compared with the healthy controls, which induced significant differences in the ratio of CD4+ T cells/ CD8+ T cells.

Research showed that CD8+ T cells were abnormally activated, having a prominent role during the process of $\mathrm{HLH}$ disease (19). Thus, the results showed that the disease was caused not only by reducing CD4+ T cells but also through an imbalance between CD8+ T-cell activation and inhibition.

It has been shown that CD4+CD25+ T cells suppress CD8+ $\mathrm{T}$-cell proliferation and interferon- $\gamma$ production induced by polyclonal or Ag-specific stimuli (20). Prominent but phenotypically variable CD8 $+\mathrm{T}$ cells were often present and were efficiently activated, particularly in EBV-associated $\mathrm{HLH}$, and increased the number of CD8+ T cells that were reported before (21); however, we did not found obvious changes in this study. A strong expression of HLA-DR was seen in most cases of EBV-associated HLH in our results. The expression of HLA-DR on granulocytes has been correlated with high levels of cytokines, including IL-2 and interferon- $\gamma$. Thus, it may serve as a marker of cytokine release (22). Cytokine storm was known as one of the important causes of death of the EBV-HLH patients. The research found that the decreased percentage of CD4+CD25+ T cells and the increased ratios of CD8+HLA-DR+ $\mathrm{T}$ cells/CD4+CD25+ $\mathrm{T}$ cells in EBV-HLH patients had a relation with death. Thus, it is concluded that the excessive release of cytokines, such as interferon- $\gamma$, resulting from the imbalance of activation and inhibition of $\mathrm{T}$ cells is one of the most important causes of death in EBV-HLH patients.

HLH in the context of infection is best described as part of a spectrum of EBV-associated illness that results in clonal proliferation of $\mathrm{T}$ lymphocytes (9). This syndrome may be difficult to distinguish from other infectious illnesses, including sepsis, and recognizing this condition is very important. We found that there were significant differences in CD8+HLA-DR+ T cells and CD8+HLA-DR+ T cells/CD4+ CD25+ $\mathrm{T}$ cells between EBV-HLH patients and the sepsis group. HLH patients exhibited a higher percentage of CD8+ HLA-DR+ T cells, and the results suggested that increased T-cell HLA-DR expression was associated with HLH patients, which indicated that antigen expression on the surface of $\mathrm{T}$ cells may be a useful tool for predicting and identifying HLH from sepsis. We evaluated the usefulness of the above indicators for the diagnosis of HLH patients. The results showed that the ratio of CD8+HLA-DR+ T cells/CD4+CD25+ $\mathrm{T}$ cells was a preferable diagnosis marker of EBV-HLH.
Finally, our novel finding of the association between excessive CD8+ T-cell activation and the decreased number of CD4+ $\mathrm{T}$ cells suggests that both the quantity and the activation conditions of $\mathrm{T}$ cells may have value for the management of patients with HLH. The ratio of CD8+ HLA$\mathrm{DR}+\mathrm{T}$ cells/CD4+CD25+ T cells may be an indicator for the diagnosis of EBV-HLH. The percentage of CD4+CD25+ $\mathrm{T}$ cells and the ratios of CD8+HLA-DR+ T cells/CD4+CD25+ $\mathrm{T}$ cells in EBV-HLH patients had a relation with the prognosis of the disease. Currently, flow cytometry is widely used for clinical diagnosis, and these specimens were easily extracted from peripheral blood cells. Therefore, this discovery may present a novel application of flow cytometry in EBV-HLH. These findings, along with clinical and laboratory information, may be used for diagnosis and differential diagnosis of HLH patients.

\section{ACKNOWLEDGMENTS}

We thank the children and parents who participated in this study for their time and generosity.

\section{STATEMENT OF FINANCIAL SUPPORT}

This project was supported by grants from the National Natural Science Foundation of China (Grant No. 81501760), Zhejiang Provincial Natural Science Foundation of China (Grant No. LQ16H050002), and the Zhejiang Provincial Healthy Science Foundation of China (Grant No. 2015KYB191). The funders did not participate in designing or executing the study.

Disclosure: The authors declare no conflict of interest.

\section{REFERENCES}

1. Jordan MB, Filipovich AH. Hematopoietic cell transplantation for hemophagocytic lymphohistiocytosis: a journey of a thousand miles begins with a single (big) step. Bone Marrow Transplant 2008;42:433-7.

2. Ishii E OS, Imashuku S, Yasukawa $M$, et al. Nationwide survey of hemophagocytic lymphohistiocytosis in Japan. Int J Hematol 2007;86: $58-65$.

3. Imashuku S. Clinical features and treatment strategies of Epstein-Barr virus-associated hemophagocytic lymphohistiocytosis. Crit Rev Oncol Hematol 2002;44:259-72.

4. Henter JI, Horne A, Aricó M, et al. HLH-2004: diagnostic and therapeutic guidelines for hemophagocytic lymphohistiocytosis. Pediatr Blood Cancer 2007;48:124-31.

5. Palazzi DL, McClain KL, Kaplan SL. Hemophagocytic syndrome in children: an important diagnostic consideration in fever of unknown origin. Clin Infect Dis 2003;36:306-12.

6. Starska K, Głowacka E, Kulig A, et al. The role of tumor cells in the modification of $\mathrm{T}$ lymphocytes activity - the expression of the early CD69+, CD71+ and the late CD25+, CD26+, HLA/DR+ activation markers on $\mathrm{T}$ CD4+ and CD8+ cells in squamous cell laryngeal carcinoma. Folia Histochem Cytobiol 2011;49:579-92.

7. Xu XJ, Tang YM, Song H, et al. Diagnostic accuracy of a specific cytokine pattern in hemophagocytic lymphohistiocytosis in children. J Pediatr 2012;160:984-90.

8. Janka GE, Lehmberg K. Hemophagocytic lymphohistiocytosis: pathogenesis and treatment. Hemophagocytic lymphohistiocytosis: pathogenesis and treatment. Hematol Am Soc Hematol Educ Program 2013; 2013:605-11.

9. Smith MC, Cohen DN, Greig B, et al. The ambiguous boundary between EBV-related hemophagocytic lymphohistiocytosis and systemic EBVdriven T cell lymphoproliferative disorder. Int J Clin Exp Pathol 2014;7: 5738-49. 
10. Tang $\mathrm{Y}, \mathrm{Xu} \mathrm{X}$, Song $\mathrm{H}$, et al. Early diagnostic and prognostic significance of a specific Th1/Th2 cytokine pattern in children with haemophagocytic syndrome. Br J Haematol 2008;143:84-91.

11. Jordan MB, Allen CE, Weitzman S, et al. How I treat hemophagocytic lymphohistiocytosis. Blood 2011;118:4041-52.

12. van der Ven AJ, Netea MG, van der Meer JW, de Mast Q. Ebola virus disease has features of hemophagocytic lymphohistiocytosis syndrome. Front Med 2015;2:4.

13. Filipovich AH. Hemophagocytic lymphohistiocytosis (HLH) and related disorders. Hematology 2009;1:127-31.

14. Canna SW, Behrens EM. Making sense of the cytokine storm: a conceptual framework for understanding, diagnosing, and treating hemophagocytic syndromes. Pediatr Clin North Am 2012;59:329-44.

15. Rosado FG, Kim AS. Hemophagocytic lymphohistiocytosis: an update on diagnosis and pathogenesis. Am J Clin Pathol 2013;139:713-27.

16. Kasahara Y, Yachie A. Cell type specific infection of Epstein-Barr virus (EBV) in EBV-associated hemophagocytic lymphohistiocytosis and chronic active EBV infection. Crit Rev Oncol Hematol 2002;44:283-94.
17. Tang YM, Xu XJ. Advances in hemophagocytic lymphohistiocytosis: pathogenesis, early diagnosis/differential diagnosis, and treatment. Sci World J 2011;11:697-708.

18. Tincati C, Bellistrì GM, Ancona G, Merlini E, d'Arminio Monforte A, Marchetti G. Role of in vitro stimulation with lipopolysaccharide on T-cell activation in HIV-infected antiretroviral-treated patients. Clin Dev Immunol 2012; 2012:935425.

19. Humblet-Baron S, Franckaert D, Dooley J, et al. IL-2 consumption by highly activated CD8 $\mathrm{T}$ cells induces regulatory $\mathrm{T}$-cell dysfunction in patients with hemophagocytic lymphohistiocytosis. J Allergy Clin Immunol 2016;138:200-9.

20. Piccirillo CA, Shevach EM. Cutting edge: control of CD8+ T cell activation by CD4+ CD25+ immunoregulatory cells. J Immunol 2001;167:1137-40.

21. McCall CM, Mudali S, Arceci RJ, et al. Flow cytometric findings in hemophagocytic lymphohistiocytosis. Am J Clin Pathol 2012;137:786-94.

22. Das UN. Insulin in the critically ill with focus on cytokines, reactive oxygen species, HLA-DR expression. J Assoc Physicians India 2007; 55(Suppl):56-65. 